\section{Ein Leiterwiderstand im Traumseminar}

Ralf Binswanger und Jeannette Widmer (Zürich)

Zusammenfassung: Widerstände und deren Bearbeitung sind zentral im psychoanalytischen Prozess - dasselbe gilt auch für ihr Auftauchen in psychoanalytischen Seminaren. Die vorliegende Wiedergabe eines Traumseminars nach Fritz Morgenthaler zeigt, wohin es führen kann, wenn aufgrund eines unbewussten Widerstands des Seminarleiters die Assoziationen der Gruppenteilnehmer nicht gebührend berücksichtigt werden. Die nachträgliche Reflexion und Analyse durch den Leiter selbst sowie die anschliessende Bearbeitung in der Seminargruppe werden in diesem Aufsatz chronologisch dargestellt und stehen beispielhaft für den Umgang mit Widerständen zur Vertiefung des Erkenntnisprozesses. Zudem können die Vorgänge und Einfälle im Traumseminar als Widerspiegelungen der Assoziationen der Träumerin oder des Träumers zum Traum verstanden und behandelt werden.

Schlüsselwörter:Traumseminar, Fritz Morgenthaler, Widerstand, Assoziationen zum Traum

\section{Einleitung}

«Was immer die Fortsetzung der Kur hemmt, ist ein Widerstand» (Freud 1900, S. 521). Der Widerstand kann vom Patienten ausgehen, aber genauso vom Analytiker (Koellreuter, 1987). Denn Psychoanalyse ist in der Praxis ein Zweipersonen-Prozess.

Das Traumseminar spiegelt im Gruppenprozess bewusste und unbewusste Vorgänge aus einer psychoanalytischen Situation, in welcher der behandelte Traum erzählt wurde. Widerstand und Gegenwiderstand übertragen sich auf die Gruppenteilnehmerinnen und die Leiterin. Diese Spiegelung kann gleichsam «rein» sein, ohne nennenswerte Behinderung durch spezifische Übertragungsreaktionen von Gruppenteilnehmern. Das Traumseminar ermöglicht es dann - ähnlich wie in Supervisionen - diese Spiegelung aufzudecken. Der Kollege, der den Traum vorgestellt hat, kann das Seminar in eigener Verantwortung für den weiteren Prozess in der von ihm geführten psychoanalytischen Therapie nutzbar machen.
Es kommt wohl häufiger vor als wir uns bewusst sind, dass ein Widerstand welcher von ausserhalb in das Traumseminar hineingetragen wird, den Prozess behindert. Geht er von einzelnen Gruppenmitgliedern aus, ist diese Behinderung meist leicht zu beheben. Es können andere Teilnehmer sein, welche erfolgreich korrigierend eingreifen; oder es können Konfusionen im Gruppenprozess sein, welche die leitende Person erkennen und im günstigsten Fall durch geeignete Interventionen transparent machen kann.

Problematischer wird es, wenn ein solcher Widerstand aus einer unbewussten Konflikthaftigkeit der Leiterin hervorgeht. Auch dann ist es möglich, dass einzelne Gruppenmitglieder oder der Gruppenprozess als solcher korrigierend eingreifen. Das kann auch durch die Rückmeldung der Kollegin geschehen, welche den Traum vorgestellt hat. Im Fall, den wir in dieser Arbeit vorstellen, erlaubte es die Sichtung und Überarbeitung des Protokolls dem Leiter, seinen Widerstand zu erkennen und die neu gewonnene Erkenntnis den Teilnehmerinnen des Traumseminars in einem späteren Seminartermin zu vermitteln.

Diesen Prozess möchten wir im Folgenden von A bis Z nachzeichnen, denn wir sind der Überzeugung, dass solche Situationen häufig vorkommen und unvermeidbar sind. Wie in analytischen Prozessen kann es nicht darum gehen, sie unbedingt vermeiden zu wollen, sondern ihr Aufdecken und Bearbeiten als Instrument zu verstehen, um die Erkenntnis zu vertiefen.

Schliesslich soll an diesem Beispiel dargestellt werden, dass und wie die Vorgänge und Einfälle im Traumseminar als Widerspiegelungen der Assoziationen der Träumerin zum Traum verstanden und behandelt werden können.

\section{Methodisches}

Das hier vorgestellte Traumseminar ist das dreizehnte und letzte einer Serie, welche in drei fortlaufenden Semestern (Sommer 2015 bis Sommer 2016) am Psychoanalytischen Seminar Zürich unter der Leitung von Ralf Binswanger (Ralf B) stattfand. Der Traum wurde von Jeannette Widmer (Jeannette W) vorgestellt.

Für grundsätzliche Überlegungen zur Methodik des Traumseminars verweisen wir auf den zweiten Abschnitt der Arbeit Traumseminare erleben (in diesem Heft). Demnach gibt die Therapeutin nur den ihr erzählten Traum ohne weitere Informationen zur träumenden Person oder zum analytischen Prozess wieder. Im Anschluss daran bearbeitet die Gruppe den Traum, während die vorstellende Kollegin schweigt, bis sie am Ende der Gruppendiskussion ihr Feedback zur Arbeit der Gruppe abgeben darf und soll. 
Die folgende integrale Darstellung des Seminarprotokolls und die nachträglich eingefügte Analyse durch Ralf B sollten es der Leserin ermöglichen, den Prozess, den wir seinerzeit durchlaufen haben, chronologisch nachzuvollziehen: Zuerst Lektüre des Traumtextes, des Gruppenprozesses - ohne die nachträglich eingefügte Analyse - und des Feedbacks von JeannetteW. Anschliessend Re-Lektüre des Gruppenprozesses inklusive der eingefügten Nachanalyse durch Ralf B mit den in Kenntnis des Feedbacks erstellten Hypothesen. Darauf folgen ein Kommentar und theoretische Überlegungen zur Widerspiegelung der Assoziationen im Gruppenprozess.

Beim Protokoll des Traumseminars handelt es sich um eine Mitschrift der Teilnehmerin Marina Patzen. Ralf B hat in ihrem Protokoll einzelne theoretische Formulierungen so verdeutlicht, dass sie für den didaktischen Zweck verständlich sind. Anschliessend hat er seine Nachanalyse eingefügt. Das Protokoll und die Nachanalyse wurden beim Verfassen dieser Arbeit nur noch geringfügig verändert, wenn es die Verständlichkeit unbedingt erforderte.

\section{Traumseminar}

Traumerzählung

Da die vorstellende Kollegin Jeannette W den Traum, der ihr eine Woche zuvor von einer Analysandin erzählt worden war, nur noch ungenau in Erinnerung hatte, liess sie ihn sich in der letzten Stunde nochmals erzählen. ${ }^{1}$

Ich habe also geträumt, dass er einen Umzug veranlasst habe. Wir waren in einer kleinen Wohnung, ganz schäbig, irgendwo aufdem Land in der Nähe von B. Die Strasse war sehr laut und dreckig. Ich dachte, jetzt muss ich halt nach O. pendeln. Und mein Bub wird aus seinem Leben und seiner Umgebung herausgerissen. Mein Gefühl war eine grosse Ohnmacht, das hat er so bestimmt, und so eingerichtet. Dann gab es noch einige so Details, die Nachbarn waren schrecklich, es war ein komisches Umfeld, die Strasse und der Lärm haben nicht aufgehört.
Gruppendiskussion

Nachanalyse des Traumseminars durch Ralf B

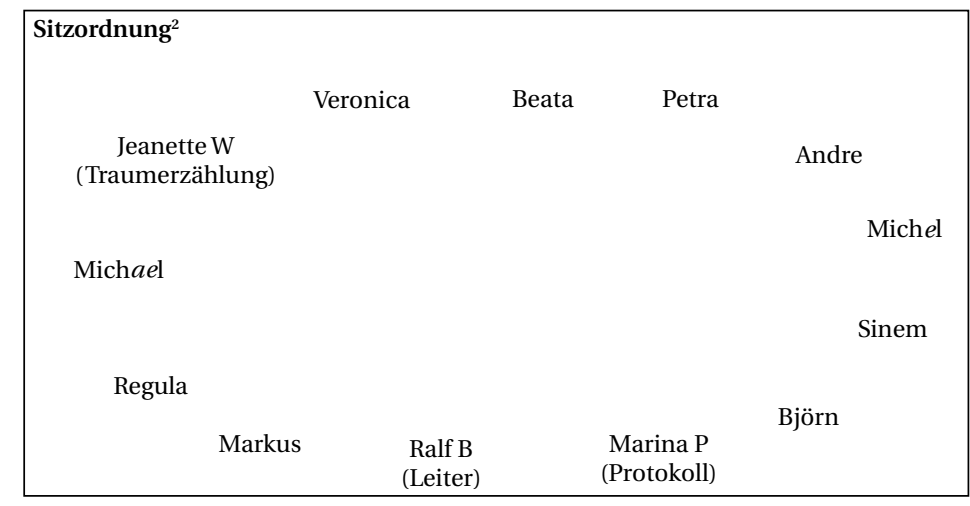

[Langes Schweigen]

Ralf B: Die Strasse ist lärmig und wir sind still.

Veronica: Ich finde es spannend, dass es mit einem Satz aufhört, der sagt, dass es nicht aufhört.

Analyse Ralf B: Die ersten beiden Interventionen enthalten in aller Klarheit eine Umkehrung.

Björn: Mir kommt das alles biblisch vor. Eine endlose Qual. Man wird eingeladen, bei der Qual zuzuschauen. Es wird genau beschrieben, wo das ist. «Er» (das, was mich so ans Biblische erinnert vom Duktus her) hat veranlasst, er hat eingerichtet, er bestimmt. Das tönt märtyrerhaft. Am Ende werden noch ein paar Details nachgeliefert, im Sinne von: Da, schaut genau hin, so schlimm ist es. Das Subjekt taucht nur in der Mitte auf in der Denkleistung (ich muss nach O. pendeln und mein Bub wird aus der Umgebung herausgerissen). Ich frage mich gerade, wer eigentlich gequält wird, ob das nicht die Therapeutin ist. Ob da nicht irgendwo eine Umkehrung ist. Ich habe nichts gefunden im Traum, das darauf hindeuten könnte. Also, dass es beim Gequältwerden eigentlich um einen Impuls zu quälen geht, der dann umgekehrt wird im Traum. 
Analyse Ralf B: Nachdem Björn eine Charakterisierung des manifesten Traumtextes als «biblisch» vorgenommen hat, kommt er auch auf die Umkehrung - ohne die Nachlieferung von Details am Ende des Traumes, die eventuell als Umkehrung aufgefasst werden könnte, als solche zu verstehen.

Ralf B: Dass sie im manifesten Traum die Gequälte ist, aber die Tendenz besteht, die Therapeutin zu quälen, einzuengen. Bei dieser Patientin hätte die Therapeutin eine enge Wohnung.

Björn: Und das hört nie auf, das geht bis ... weiss ich nicht. Endlose Höllenqualen.

Michel: In diesem Traum hat es etwas Vergleichbares mit dem Militärtraum, der in Wittmanns Artikel steht. ${ }^{3}$ Sie kann überhaupt nichts machen, sie ist nur Beobachtende und berichtet ihr Leidwesen. Das Einzige, womit man der Sache ein bisschen näherkommt, ist das Gefühl einer grossen Ohnmacht.

Ralf B: Wie eine Soldatin in Lutz Wittmanns Artikel. Es ist keine Entwicklung vom einen zum anderen und es wird besser oder schlechter, sondern es bleibt stehen. Sie bleibt gefangen im manifesten Traum. Was müsste man tun, wenn man Björn folgen wollte mit der Umkehrung?

Björn: Schauen, ob man im Traum formale Elemente der Umkehrung findet.

Analyse Ralf B: Sowohl Björn als auch Ralf B übersehen die Umkehrung in den ersten beiden Interventionen.

Ralf B: Das wäre einmal das Erste. Aber da hast du gesagt, du hättest nichts gefunden. Und was tun wir jetzt, wenn wir annehmen, es sei eine Umkehrung, auch von den Personen im manifesten Traum? Es wird die Therapeutin gequält.

Björn: Alles umkehren und konsequent ersetzen.
Ralf B: Die Therapeutin hat eine schöne, weite Wohnung, muss nicht pendeln, es geht nicht ewig weiter, sondern hat einen glücklichen Schluss ...

Analyse Ralf B: Schon hier hätte man draufkommen können, dass die beengende Qual in eine erweiternde Befriedigung umgekehrt werden könnte. Der Träumer oder die Träumerin ist «Er», der die Therapeutin befriedigen möchte. Diese dürfte passiv bleiben müsste nicht pendeln und könnte selbstbestimmt mitmachen, ohne dass «Er» das bestimmt hätte. Dann hätte alles einen glücklichen Schluss.

Ralf B: ... Wenn wir gerade daran sind, wie die Zensur arbeitet Arbeitet sie mit der Verkehrung ins Gegenteil? Es ist lustig: Überall, wo ich etwas über Träume zu erzählen hatte, sagte ich immer, dass Verschiebung, Verdichtung, Verkehrung ins Gegenteil und Symbolisierung die Hauptmechanismen der Traumzensur seien. Und vor zwei Jahren habe ich plötzlich bemerkt, dass Freud nirgends von der Verkehrung ins Gegenteil als gleichberechtigt zur Verschiebung und Verdichtung redet. Obwohl in den Träumen und überall, auch bei den Abwehrmechanismen, die Verkehrung ins Gegenteil sehr häufig ist. Man stösst durchaus immer wieder auf Träume, in denen die Zensur mit der Verkehrung ins Gegenteil arbeitet ...

Analyse Ralf B: Statt dass Ralf B seinen stimmig ansetzenden Einfall zu Ende führt, wehrt er ihn mit Intellektualisierungen ab ..

Ralf B: ... Hier würde man das vielleicht finden, wenn man die Assoziationen noch hätte, aber im Traum selber findet man nichts.

Analyse Ralf B: ...und übersieht die Umkehrung in den ersten beiden Interventionen, welche im Traumseminar ja anstelle der Assoziationen stehen - und die erste Intervention hat er erst noch selber gemacht - respektive das Schweigen der ganzen Gruppe als Umkehrung zur lärmigen Nachbarschaft interpretiert. 
Ralf B: Aber was findet man im Traum? Wenn wir es jetzt von den Abwehrmechanismen haben? Wie arbeitet die Zensur? Es ist so einfach, dass man es gar nicht merkt. Wenn man es jetzt im manifesten Traum sucht?

Veronica: Masochismus?

Markus: Hilflosigkeit.

Ralf B: Nein. Mit welchen Hilfsmitteln arbeitet die Zensur? Das war meine Frage, wenn wir schon an diesem Thema sind, oder? Mit der Verschiebung. Von O. nach B. Wer macht das? Der Traum. Wenn man konsequent überlegt, wie die Zensur arbeitet, kommt man viel einfacher auf die Verschiebung als auf die Verkehrung ins Gegenteil.

Analyse Ralf B: Die intellektualisierende Abwehr von Ralf B hat sich offensichtlich verfestigt.

Markus: Bei mir ist es als ein passiv-aggressives Lamento angekommen.

Veronica: Für mich hat es etwas Sadomasochistisches darin gehabt. Dass sie ein Stück weit geniesst, dass er so über sie verfügt und sie erniedrigt wird in dieser kleinen Wohnung.

Analyse Ralf B: Die letzten beiden Interventionen versuchen, das zu korrigieren, was aber deshalb misslingt, weil sie sich am manifesten Trauminhalt orientieren. Das passiv-aggressive Lamento könnte in ein aktiv libidinöses Verführen umgekehrt werden, und das Geniessen wäre dann zwar angemessen, müsste aber in allen Aspekten umgekehrt werden: «Er» geniesst es, einvernehmlich die aktive Rolle zu übernehmen, was für «Sie» - die Analytikerin erhebend neue Räume eröffnet.

Björn: Man soll verführt werden zum Lusthaben am Zuschauen. Vom Klima her, oder?
Analyse Ralf B: Björn erkennt das jetzt, verschiebt aber die passivlustvolle Beteiligung aufs Zuschauen - was eventuell auch wieder eine Umkehrung sein könnte.

Michael: Mich erinnert der Traum an eine Filmszene, einen HorrorRoad-Trip von O. nach B.

Ralf B: Und Schwupps sind wir wieder beim Inhalt des manifesten Traums. Aber wenn wir beim Formalen bleiben: Was wird verschoben? Ein Element des Traums. Das Kind z.B. oder vom «Er» zur Therapeutin. Das Kind wird zwar im manifesten Traum auch verschoben. Aber es könnte sonst ein Element sein, das von irgendwo anders her in diesen Traum hineinverschoben wird. Und dann im Traum verpackt wird und man merkt es nicht. Wenn die Zensur mit der Verschiebung arbeitet.

Analyse Ralf B: Ralf B verharrt in seiner Abwehr, obschon es naheliegend wäre, draufzukommen, dass «Er» der Therapeutin ein Kind machen will.

Andre: Mir kommt gerade der Gedanke wegen dieses Lamentos. Ich fand das eben auch so. Ich fand das so ganz ich-synton. Und dann kam so die Vorstellung, die Träumerin ist depressiv. Und was verschoben wird, das ist diese Aggression gegen sich selbst. Die Aggression habe ich sehr empfunden und der Hass ist auch gut spürbar in den Formulierungen. «Er» hat das so bestimmt. Schon im ersten Satz wird definiert, wer der Quäler ist, also er. Sonst, im realen Leben, ist es immer gegen sich selbst. Und im Traum, da wird einmal ein Schuldiger definiert.

Analyse Ralf B: Die Orientierung am manifesten Trauminhalt verhindert erneut, die Umkehrung vollständig zu vollziehen: Zwar wird die Depression = Aggression gegen sich - der Richtung nach umgedreht zum Hass auf den «Er», aber nicht der Inhalt der Emotion, vom depressiven Tief zur phallischen Hochstimmung, der Hass nicht zur Liebe, und auch nicht die Personenzuteilung, 
also «Er» im manifesten Trauminhalt $=$ «Sie», die Träumerin, im latenten Traum.

Ralf B: Also die Aggression wird verschoben von ihr zum «Er». Andre denkt an eine depressive Patientin, die gewohnheitsmässig immer zu kurz kommt.

Markus: Und auf das Subjekt wütend ist, aber das nicht richtig weiss. Beim Ich-synton bin ich im Zweifel. Für mich hat es kein depressives Element, sondern etwas Gespaltenes. Die Abwehr könnte eventuell unreifer sein als die Verschiebung.

Analyse Ralf B: Intuitiv schaltet Markus mit der Feststellung, es gebe kein depressives Element, adäquat. Im Sinne der Umkehrung könnte er aber statt «unreifer», «reifer» sagen, was dann heissen könnte: Statt Verschiebung - charakteristische Abwehr der frühphallischen Phase - aktives Agieren - charakteristisch für die spätphallische Phase. ${ }^{4}$

Björn: Wir haben ja gesagt, die Traumtendenz, die lässt sich ja auch manchmal erkennen. Wie wird ein Traum in welchem Kontext erzählt? Der Traum wird zum zweiten Mal erzählt. Es gibt eine voyeuristische Position, sie will es nochmals hören. Ich komme nicht davon weg, dass da etwas bei der Zuhörerin oder den imaginierten Zuhörern bewirkt werden soll ...

Analyse Ralf B: Das würde die hier erarbeitete Hypothese vollkommen treffen: Eine reale Befriedigung der Analytikerin bewirken was einem unbewussten Agieren in der Übertragung entspricht.

Björn: ... Dass abgelenkt werden soll von etwas. Der Traum wird erzählt, dann kommt die Denkleistung, wie abgesetzt davon. «Ich habe also geträumt.» Danach das Martyrium. Im Anschluss: «Also dachte ich.» Und dann das Gefühl der grossen Ohnmacht. Und anschliessend könnte man sagen, es ist fertig. Aber es gibt noch Nachschlag: «Hier noch einige Details.» Und das Ganze hört nie auf. Die Tendenz der Erzählung: Es soll auf etwas gelenkt werden, damit man etwas Anderes nicht sieht. Ich habe ganz am Anfang gedacht, vielleicht geht es einfach um eine Zahlung, die nicht veranlasst wurde. Umzug veranlassen bedeutet Zahlung veranlassen. Vielleicht hat die Patientin nicht bezahlt. Verschiebung sieht man ja manchmal in Wörtern.

Analyse Ralf B: Da ist die Umkehrung: «Er hat die Zahlung veranlasst» $\mathrm{zu}$ «Sie hat die Zahlung nicht veranlasst.» Wenn «Er» die Analytikerin zur heterosexuellen Geliebten machen könnte - was leider für «Sie», die Träumerin, ganz unmöglich ist - dann müsste sie ihr auch keine Analysestunden bezahlen ...

Markus: Oder ein endloser Ehekonflikt.

Analyse Ralf B: ... oder es müsste dann bei der Scheidung bezahlt werden.

Björn: Aber das wäre alles zu nahe am manifesten Traum.

Ralf B: Eine gewagte Hypothese, so lernt man aber, aus den Träumen etwas zu machen. Ich habe mir überlegt, während du geredet hast: Man könnte natürlich den Nachtrag als Umkehrung betrachten. Sie kommt nochmals darauf zurück. Das könnte die Umkehrung sein.

Analyse Ralf B: Björn hilft Ralf B, aus seiner Abwehr herauszukommen - was allerdings nur vorübergehend gelingt, denn Ralf $\mathrm{B}$ kommt gleich wieder von der Umkehrung weg und intellektualisiert weiter.

Markus: Es ist wie ein in sich abgeschlossener Artikel, eigentlich sehr medial präsentiert.

Ralf B: Um noch einmal darauf zurückzukommen: Deine [Björns] Hypothese ist, was Morgenthaler die elliptische Auslassung nennt. Die Ellipse hat ja zwei Brennpunkte. Und indem man ständig den einen dieser beiden Brennpunkte berücksichtigt, wird das, worum es eigentlich geht, ausgelassen. Das ist auch eine Form von 
Verschiebung. Ich habe Morgenthaler so verstanden. Es gibt einen blinden Fleck ...

Analyse Ralf B: Ralf B spricht von dem, was er selbst hat: einen blinden Fleck, ein Skotom im Gesichtsfeld nämlich für die Umkehrung in den beiden ersten Interventionen des Gruppenprozesses.

Ralf B: ... Man müsste von dem, was suggeriert wird in dieser Intensität, absehen und schauen, wo es eine Leerstelle gibt.

Wir haben zwei verschiedene Tendenzen: das Passiv-Aggressive, das Traumatische, die Spaltung. Das Traumatische ist oft näher am manifesten Trauminhalt. Die andere Tendenz: Sie ist depressiv, eine progressive Tendenz im Traum, die Aggressionen können mal raus. Vorher haben wir nach Abwehrmechanismen gesucht, jetzt denken wir strukturell. Spaltung versus Depression.

Markus: Ich habe eine grundsätzliche Frage. Ich weiss ja jetzt, wie diese Seminare gehen. Aber für mich ist es immer relevant für ein besseres Traumverständnis, zu erfahren, was der Träumer assoziiert zum Traum.

Analyse Ralf B: Markus versucht, uns zu den ersten Interventionen zurückzubringen, welche im Traumseminar ja anstelle der Assoziationen in der Behandlungssituation stehen, ...

Regula: Morgenthaler fand, man erhalte nur Rationalisierungen, wenn man assoziieren lässt.

Analyse Ralf B: ... scheitert aber damit, weil Regulas Intervention Ralf B zum weiteren Rationalisieren verführt, ...

Ralf B: Morgenthaler meinte, es sei aufschlussreicher, alles, was in der Stunde der Traumerzählung passiert, so aufzufassen, dass dies die Assoziationen dazu sind.
Markus: Und wenn nach dem erzählten Traum nichts passiert? Dann warten wir einfach?

Ralf B: Dann schauen wir, was vor der Erzählung alles gesagt wurde. Alles, was gesagt wurde, ist bereits eine Assoziation zum Traum. Morgenthaler meinte, was vor der Traumerzählung kommt, sei besonders relevant im Zusammenhang mit dem Traum. Die bewusst beigebrachten Assoziationen würden unter dem Einfluss derselben Zensur stehen, wie der Traum selbst. Man bekommt vorwiegend lediglich Rationalisierungen. Das ist neu im Vergleich $z u$ Freud. Im Psychoanalytic Quarterly (Binswanger, 2016) habe ich geschrieben, dass es zwei zentrale Innovationen sind, die Morgenthaler gemacht hat. Erstens hatte er eine völlig andere Auffassung bezüglich der Assoziationen zu Träumen, und zweitens führte er die Traumdiagnostik ein als der Teil beim Umgang mit dem Traum, der mit Übertragung und Gegenübertragung oder mit abgewehrten Inhalten aus der Vergangenheit zu tun hat ...

Analyse Ralf B: ... so dass der Leiter schliesslich glaubt, mit seinem Erfolg beim Psychoanalytic Quarterly auftrumpfen zu müssen.

Ralf B: ... Und damit haben wir eine Lösung für diese Dinge, welche in der Traumdeutung von Freud nicht zu finden sind, weil es 1900 die Übertragung noch gar nicht gegeben hat, weil Freud diese erst 1905 im Fall Dora entdeckt hat. Und zur Gegenübertragung gibt es höchstens Spuren bei ihm, sie ist dann später dazugekommen. Aus der Traumdeutung alleine kann man gar nicht sinnvoll den Umgang mit Träumen ableiten. Es braucht noch mehr. Und Freud selbst hat einen ganz wichtigen Teil geleistet, noch im Abriss der Psychoanalyse, 5. Kapitel, als er die Instanzenlehre und Strukturtheorie auf die Traumdeutung angewendet hat. Morgenthaler meinte, Freud habe das nicht gemacht, und gesagt, Freud habe vergessen, die Strukturtheorie auf den Traum anzuwenden. Weil er den Abriss nicht gelesen oder wieder vergessen hat. Deserno (1999) hat nicht den Abriss genommen, um eine neuere Entwicklung von Freud zu dokumentieren (er hat ja so eine Anthologie gemacht mit verschiedenen Texten), sondern das Kapitel 
aus der Neuen Folge der Vorlesungen, wo Freud sagt, da sei nicht viel Neues drin. Das wirklich Neue ist im Abriss drin. Lansky (1992) hat eine ganz ähnliche Anthologie schon früher gemacht, und auch er hat das Kapitel aus der Neuen Folge abgedruckt. Es gibt bei den meisten psychoanalytischen Autorinnen und Autoren so etwas wie ein «Skotom» für das Traumkapitel im Abriss. Und dann gibt es drei, vier Autoren, die dieses Kapitel genau andersrum verstanden haben. Sie sagen, im Abriss habe Freud die Wunscherfüllungstheorie verlassen. Die Wunscherfüllungstheorie betrifft jedoch das Resultat und nicht den Anfang der Traumarbeit. Im Abriss nennt Freud das, was am Anfang der Traumarbeit stehen kann, statt «Wunsch» einen "Anspruch aus dem Es an das Ich auf Befriedigung eines Triebes». Das kann ein schlafstörender Reiz sein. Daneben gibt es schlafstörende Reize in Form von etwas, was einen im Wachleben beschäftigt. Und dann zitiert einer dieser Autoren, Blechner, sogar falsch: Er zitiert aus Dora, wo auch Freud gesagt habe, es sei nicht immer ein Wunsch. Und wenn man diese Stelle nachschaut, sagt Freud: «Ich hätte es leichter, wenn ich sagen würde, es sei nicht immer ein Wunsch, sondern auch mal eine Befürchtung oder das oder jenes ...»

Analyse Ralf B: Wobei er dann das Skotom bei psychoanalytischen Autoren sieht, statt dasjenige, das er selbst hat: das Skotom für die Umkehrung, welche bereits in den ersten beiden Interventionen klar wird, ...

Ralf B: Die Freudtexte, das ist schon Material, das mit dem Unbewussten arbeitet, da muss man sich nicht wundern, wenn wir voller Fehlleistungen sind, wenn wir uns damit auseinandersetzen.

Analyse Ralf B: ... wofür er um eine milde Beurteilung für seine Fehlleistung bittet.

Ralf B: Entschuldigung, vielleicht hat mein langer Sermon etwas mit der Patientin zu tun: Man geht immer weg von ihr.

Björn: Ja, wir sind wieder bei Skotomen und bei Umkehrungen.
Ralf B: Jawohl. Und das Skotom, das ist eine Patientin, der es vielleicht oft passiert, dass andere Sachen interessanter sind als sie. Und im Traumseminar, wo wir die Assoziationen nicht haben, benutzen wir die spontanen Assoziationen der Gruppenmitglieder.

Analyse Ralf B: Wenn Ralf B es nur konsequent täte.

Björn: Vielleicht versuchst du nun, uns so zu füttern, mit so umfangreichem Material, wie die Patientin dies mit der Therapeutin gemacht hat. Mit so einer angereicherten Geschichte.

Ralf B: Nachdem sie aufgefordert wurde: «Erzähl nochmal.» Man könnte es auch umkehren. Jeannette $W$ hätte auch aus ihren Erinnerungen den Traum bringen können, aber sie liess sich den Traum noch einmal erzählen. Möglicherweise dachte sie, dass das, was sie noch erinnert hat, zu wenig sei. Das wäre wieder diese Bewegung, welche der Patientin passiert, man geht von ihren Sachen weg. Man könnte dann auch sagen, wir seien den Inhalten des Traumes, welche die Patientin beschäftigen, ausgewichen, indem wir über Abwehrmechanismen theoretisiert haben.

Analyse Ralf B: Es gelingt Ralf B immer wieder nur halbwegs, seine Abwehr aufzuheben.

Michel: Ist das nicht auch eine Form von projektiver Identifikation? Es könnte ja auch wie ein Coping-Versuch sein, in den engen Raum, den wir da haben, Leben hineinzubringen.

Ralf B: Das könnte man als projektive Identifikation verstehen oder als Befreiungsversuch aus diesem engen Raum. Das heisst, sie schafft es nicht einmal, die Leute in die projektive Identifikation hineinzubringen, die gehen immer alle gleich wieder.

Regula: Gut, aber vielleicht weigert man sich auch irgendwo ein bisschen, dieser Aufforderung nachzukommen, sie vor dem «Er» zu retten, der sie unter Druck setzt, zwingt und ohnmächtig macht. Das ist wie eine fehlende Triangulierung, wo sie versucht, einen 
Dritten hereinzuholen, der ihr helfen soll. Aber man merkt, dass man hier nicht mit hineingezogen werden möchte.

Ralf B: Oder es ist wirklich etwas Traumatisierendes dabei, zum Beispiel eine traumatisierende Paarbeziehung.

Michel: Über eine Person haben wir jetzt gar nicht geredet, nämlich über den Buben. Das irritiert mich ein wenig. Der geht einfach verloren.

Björn: In der Traumerzählung ist mir der Bub vorgekommen wie eine Erweiterung ihrer selbst. Wie ich und mein Fuss. In der Denkleistung, das war ja die einzige Denkleistung.

Ralf B: Die Denkleistung wäre der latente Traumgedanke, also die Wunscherfüllung. Mein Bub wird aus seinem Leben und seiner Umgebung gerissen. Diese Denkleistung als Wunscherfüllung zu verstehen wirkt merkwürdig, es funktioniert nicht wirklich. Und der Bub ist im Prozess tatsächlich verlorengegangen.

Markus: Vielleicht ist der Träumende ein «Er» und nicht eine «Sie». Vielleicht eine traumatische Sequenz aus der Kindheit von einem enorm dominanten Vater.

Björn: Ich bin vielleicht etwas eingeengt heute, aber ich denke, es ist ein Angebot zur Unterwerfung, die man nicht haben will, womit wir gefüttert werden sollen. Vielleicht will sie uns füttern, damit sie interessant ist. Ich habe überlegt, ist das ein schwuler Mann in einer homoerotisch unterwerfenden Position? Eben mit dieser SM-Geschichte.

Markus: Oder ein umgebauter Mann.

Björn: Ein Etikettenschwindel. In der Dose ist nicht ganz das drin, was draufsteht. Oder es ist genau das. Genau das Traumatische, was erzählt wird. Manifest = latent, dann wären wir jetzt die wegschauenden Dritten.
Die Ideen sind nicht schlecht, denn man könnte das «Queere» dieser Einfälle auf das «Queere» der aktualisierten Übertragung beziehen: Die starke männliche Seite der Träumerin versucht sich in der heterosexuellen Verführung der Analytikerin, was ganz konkret nur ginge, wenn sie ein umgebauter Mann wäre. Und auch dann gäbe es keinen Buben, also kein Kind. Der Bub würde nicht aus dem Leben gerissen, sondern man könnte kein Mädchen ins Lebendige der Analytikerin hineintun.

Ralf B: Oder der verlorengegangene Bub wäre das andere Zentrum in der Ellipse. Der Bub wird hereingeholt in den Traum.

Analyse Ralf B: Das würde auf den hier als richtig angenommenen Weg zurückführen: Die Umkehrung ist ja, dass nicht ein Bub verloren geht, sondern ein Mädchen zur Welt kommen wird. Der hier als richtig angenommene Weg könnte also zu folgender Hypothese führen: Die Traumerzählung aktualisiert einen Anspruch an das Ich, innerhalb der Übertragung einen männlich-phallischen Trieb zu befriedigen und der Analytikerin ein Mädchen zu machen = sie zu schwängern. Dieser Anspruch muss aus zwei Gründen abgewehrt werden:

1. weil sie mit der Analytikerin ja eine Analyse vollziehen will und nicht einen Geschlechtsverkehr;

2. weil sie mit diesem Triebanspruch als Frau erneut ins Leere fallen müsste, da sie ja nicht mit einem Penis ausgestattet ist, um einen aktiv-genitalen Vollzug zu bewerkstelligen. Um weiterschlafen zu können, muss also dieser Triebanspruch durch einen harmlosen latenten Traumgedanken ersetzt werden: «Jetzt muss ich halt nach O. pendeln, und mein Bub wird aus seinem Leben und seiner Umgebung herausgerissen.» Die Traumtendenz aktualisiert also einen Übertragungskonflikt, der gedeutet werden kann und soll.

Björn: In der Denkleistung.

Michael: Es reden fast nur Männer in unserer Gruppe. Man übergeht die Person vielleicht, indem man die ganze Zeit über das Traumatische redet, hat das nicht auch etwas Retraumatisierendes? 
Ist nicht auch etwas sehr Brutales in dieser Bewegung drin? In der Analyse würde man das ja nicht machen.

Ralf B: Klar nicht, wir haben hier eine didaktische Veranstaltung, keine Supervision. Wir üben, wie man mit Träumen umgehen kann ...

Analyse Ralf B: Michael reagiert wohl adäquat auf die brutale Abwehr von Ralf B, welcher seine Intervention deshalb brutal abwehren muss, ...

Ralf B: ... Ich komme nicht weg davon, zu fragen, ob sie eine dieser Menschen ist, die so fest appellieren und etwas auslösen müssen mit einer oberflächlich zwar guten Abwehrstruktur. Der Traum ist ja gut strukturiert, einfach ein wenig zu eng. Und dann wird noch dieses und jenes hinzugefügt. Die vielen Nachbarn haben wir auch noch, die haben wir auch vergessen. Aber es wäre jemand, der ständig Gefahr läuft, dass man nicht bei ihm/bei ihr bleibt.

Michael: Das ist das, was bei mir Unbehagen auslöst. Wenn wir so theoretisieren, gehen wir auch weg von ihr. Aber das ist die Veranlagung unseres Settings.

Analyse Ralf B: ... dabei hat Michael bezüglich des heutigen Traumseminars vollkommen Recht. Es liegt allerdings nicht am Setting, dass Ralf B so stark ins Theoretisieren kommt, sondern an seinem unbewussten Widerstand.

Markus: Vielleicht gibt es ganz viele Verschiebungen. Kommt mir im Zusammenhang mit dem Traumatischen in den Sinn. Der Lärm und die Strasse, die nie enden, sind eventuell eine Verschiebung auf reale, nie endende schwere Konflikte. Im Schäbigen drin habe ich vor allem das Passiv-Aggressive gesehen. Dort drin hat es auch etwas, was appellativ ist.

Ralf B: «Du musst mich aus dem Schäbigen rausnehmen»: eine Retterfantasie.

\section{Feedback der Therapeutin Jeannette W}

Die Träumerin ist eine Frau, 38 Jahre alt. Sie kommt seit sieben Monaten zweimal dieWoche liegend in die Analyse. Sie ist in einem Masterstudium, arbeite aber Teilzeit. Sie hat einen 8-jährigen Sohn und ist tatsächlich mit der Beziehung sehr beschäftigt. Sie leidet darunter, dass mit ihrem Ehemann wenig emotionaler und intellektueller Austausch möglich ist.

Sie kam in die Analyse, wollte sich mit sich selbst beschäftigen, sich beruflich entwickeln und wurde unerwartet schwanger. Das riss ihr den Boden unter den Füssen weg. Sie erzählte es dem Mann zuerst nicht und machte mit sich selber ab, ob sie das Kind behalten wolle.

Sie entschied sich für das Kind, da sie sich eigentlich ein zweites Kind wünschte. Der Mann freute sich sehr, als sie es ihm sagte. Er stellte darauf ihre Sachen um in der Wohnung, was sie ärgerte.

Für das erste Kind wünschte sie sich eine Hausgeburt, die dann aber nicht gut verlief. Der Mann ging weg, trank zu viel, und sie fühlte sich alleine gelassen. Da merkte sie, dass er ein Alkoholproblem hatte. Erst vor drei Jahren stellte sie ihm ein Ultimatum, dass sie gehe, wenn er nicht aufhöre zu trinken. Daraufhin hat er aufgehört. Er sei immer ein liebevoller und engagierter Vater dieses Knaben gewesen. Dem Traum voraus ging der Vorschlag des Mannes, dass sie Karriere mache und er Hausmann werde. Den Traum hatte sie Ende April erzählt, da war sie etwa in der 30. Schwangerschaftswoche.

Dann noch zur Zahlung, das fand ich interessant. Sie möchte über die Zusatzversicherung abrechnen. Ich habe ihr schon zwei Rechnungen gegeben, sie hat diese auch der Zusatzversicherung weitergereicht, aber diese wollen zuerst einen Bericht. Die Zahlung war, als sie den Traum erzählte, tatsächlich noch ausstehend, und die Analysandin fragte nach, ob sie noch zuwarten könne.

Das Manifeste hat ganz viel zu tun mit ihrem Mann. Es ist eine schwierige ungleiche Beziehung. Aber er ist der Vater ihrer Kinder und sie hat sich mit ihm arrangiert. Sie wurde mit einer Hüftgelenkluxation geboren und lag viele Monate im Gips. Das ist möglicherweise das Eingeengtsein.

\section{Kommentar}

Im Rückblick auf dieses Traumseminar und den Gruppenprozess ist es beeindruckend, wie die Assoziationen in der Gruppe mehrfach auf den Widerstand des Leiters aufmerksam machten, dieser jedoch erst bei der nachträglichen Reflexion bewusst wurde. Möglicherweise spiegelt dies einen unbewussten 
Widerstand der Analytikerin in Bezug auf das triebhafte Übertragungsgeschehen ihrer Analysandin

Der Widerstand des Leiters zeigte sich am deutlichsten an seiner «Blindheit» gegenüber der naheliegenden Hypothese, dass die Traumzensur mit dem Mechanismus der Verkehrung ins Gegenteil arbeitete. Erst das konsequente Umkehren aller Inhalte in der Nachanalyse brachten den Leiter zu der Hypothese, dass die Traumerzählung einen Anspruch des Es an das Ich aktualisiert, innerhalb der Übertragung einen aktiv-phallischen Triebwunsch zu befriedigen: «Er» will ihr ein Kind machen - von der beengenden Qual zur erweiterten Befriedigung. Vermittelt über den «schwangeren Bauch» ist es der Analysandin gelungen, einen aktiv-phallischen Wunsch in der Übertragung unterzubringen, nämlich den im Unbewussten ganz konkreten Wunsch, sie zu penetrieren und dadurch zu schwängern. Darauf reagierte die Analytikerin mit dem hier dargestellten Widerstand.

Die Schwangerschaft wurde von der Therapeutin zwar vorgängig als «weibliche Potenz» gedeutet. Die nachfolgende Traumerzählung könnte man demnach als Dank der Analysandin für diese Deutung auffassen. Allerdings konnte das triebhafte Geschehen innerhalb der Übertragung vor dem Traumseminar nicht berücksichtigt werden. Das mutigere Deuten der Triebwünsche unter Berücksichtigung des Konzeptes der «psychischen Bisexualität» (Gsell \& Zürcher, 2011) war denn auch für die den Traum erzählende Kollegin das wesentliche Moment, das sie aus dem Traumseminar für die weiterführende Analyse mitnahm.

Wie bereits erwähnt, kann es in analytischen Prozessen nicht darum gehen, Widerstände und Gegen-Widerstände (Koellreuter, 1987) vermeiden zu wollen, sondern die emotionalen Bewegungen aufzudecken und zu bearbeiten, welche zu Widerständen geführt haben. Gelingt dies, werden sich der analytische Prozess vertiefen und die Erkenntnisse erweitern.

\section{Ein Stück Theorie: Die Assoziationen widerspiegeln sich}

\section{im Gruppenprozess}

An unserem Beispiel lässt sich exemplarisch aufzeigen, wie produktiv es sein kann, im Traumseminar den Gruppenprozess zu beachten und in die Bearbeitung des vorgestellten Traumes einzubeziehen. Zum Gruppenprozess gehört alles, was von den Teilnehmerinnen, aber genauso vom Leiter, verbal und nonverbal eingebracht wird, und zwar vom allerersten Moment an. Das aussergewöhnlich lange Schweigen nach der Traumerzählung steht im Kontrast zum Lärm auf der Strasse, welcher den manifesten Traumbericht abschliesst. Zwar spricht der Leiter diese Umkehrung an, würdigt sie aber zu wenig, so dass er sie wieder aus den Augen verliert. Anschliessend findet es eine Teilnehmerin spannend, dass der Traum mit einem Satz aufhört, der sagt, dass es nicht aufhört - ebenfalls eine Umkehrung

Die Fokussierung auf die Phänomene des Gruppenprozesses widerspiegelt die Fokussierung auf alles, was von Analysandinnen, aber genauso von Analytikerinnen, verbal und nonverbal eingebracht wird, und zwar vom ersten Moment einer Sitzung an, in der ein Traum erzählt wird. Die Tatsache, dass wir hier zwanglos die gleiche Formulierung brauchen können wie oben, verweist auf das, worauf wir hinauswollen. Die Formulierung entspricht nämlich etwa dem, was Morgenthaler (1986, S. 24) bereits von Freud aufgreift und dann als innovativen Vorschlag bezüglich der Assoziationen zum Traum macht: Man soll Träumer nich «assoziieren lassen». «Damit sage ich nicht, dass wir auf die Assoziationen des Träumers verzichten können. Wir sind in hohem Grad auf sie angewiesen» (1986, S. 80). Übersetzt heisst das: Nicht der Analysand oder die Analysandin müssen lernen, zu ihren Träumen zu assoziieren, sondern wir müssen lernen, seine oder ihre verbalen und nonverbalen Beiträge als Assoziationen aufzufassen und damit zu arbeiten. In der allgemeinen psychoanalytischen Technik entspricht das der Sukzession im Assoziationsverlauf (Morgenthaler, 1978, S. 30-41); in der Technik des Umgangs mit Träumen heisst es: Wir haben es in der Analyse der Träume von Patienten leichter als Freud in der Analyse seiner eigenen Träume, denn die Assoziationen sind schon da.

Auch im Traumseminar sind wir «in hohem Grad» auf Assoziationen angewiesen, obschon wir diejenigen der Träumer gar nicht kennen. Wir entnehmen sie dem Gruppenprozess, den wir als Widerspiegelung der Assoziationen der Träumerinnen auffassen. Wie und warum kann das überhaupt derart exakt funktionieren, wie es in unserem Traumbeispiel offenbar der Fall war?

Eine mögliche Antwort darauf gibt z. B. Wolfgang Leuschner (2005/2006). Er stützt sich dabei auf experimentelle Untersuchungen im Sigmund Freud-Institut Frankfurt a. M. Diese konnten zeigen, dass Bilder, welche Versuchspersonen während des Bruchteils einer Sekunde (z. B. 10-20 Millisekunden) präsentiert wurden später in Zeichnungen ihrer Träume wieder auftauchten. Etwas Ähnliches konnte über tachyakustische Reize erreicht werden: Sie bestanden aus wenigen Sätzen welche den Versuchspersonen in zweieinhalbfacher Geschwindigkeit vorgelesen wurden, so dass sie bewusst nicht verstanden werden konnten. ${ }^{6}$ Leuschner ging noch einen Schritt weiter, indem er anscheinend auch telepathische Vorgänge experimentell nachwies. Seine zusammenfassende Schlussfolgerung lautet: 
Morgenthalers Behauptung, dass latente Traumgehalte allein aus manifesten «nackten» Traumerzählungen erschlossen werden können, lässt sich stützen, wenn man unterstellt, dass das Latente «subliminal» immer auch mitvermittelt und von Traumgruppen dann assoziativ erschlossen werden kann. (Leuschner, 2005/2006, S. 63)

Damit schliesst sich der Kreis zum Beginn des Gruppenprozesses: Die beiden ersten Interventionen enthüllten, gemäss unserer Hypothese, präzise, mit welchem Hauptmechanismus die Traumzensur arbeitete: mit der Umkehrung, der Verkehrung ins Gegenteil. Das unbewusste Ich, welches die Traumzensur ausübt, ist dann konsequent: Alle formalen und inhaltlichen Elemente des Traumes werden umgekehrt. Die Analyse muss demnach alles erneut umdrehen, um zur Aufdeckung nicht nur der latenten Inhalte des Traumes, sondern auch der Traumtendenz vorzudringen. ${ }^{7}$

\section{Schluss}

Das Traumseminar ist in erster Linie ein didaktisches Unterfangen. Es kann eine Supervision nicht ersetzen, weshalb die vorstellende Kollegin in ihrer weiteren Arbeit mit dem Träumer kritisch überprüfen soll, ob die im Seminar entwickelten Hypothesen für den analytischen Prozess brauchbar sind.

Wir haben dieses Beispiel ausgewählt - einerseits, weil es sehr schön zeigt, wie sich die fehlenden Assoziationen durch die Interventionen im Gruppenprozess widerspiegeln. Andererseits illustriert es etwas Alltägliches, nämlich das Auftreten von Widerständen auch im Traumseminar. Je klarer das bewusst wird, desto eher gelingt es, den Widerstand aufzudecken und zu bearbeiten.

\section{Literatur}

Binswanger, R. (2016). Dream Diagnostics: Fritz Morgenthaler's Work on Dreams.

The Psychoanalytic Quarterly 85 (3), 727-757.

Deserno, H. (1999). Das Jahrhundert der Traumdeutung. Stuttgart: Klett-Cotta.

Freud, S. (1900). Die Traumdeutung. GW II/III.

Gsell, M. \&Zürcher, M. (2011). Licht ins Dunkel der Bisexualität. Bisexualität, anatomische Geschlechtsdifferenz und die psychoanalytische Bedeutung von «männlich» und «weiblich». Psyche-Zeitschrift für Psychoanalyse und ihre Anwendungen 65, 699-729.

Koellreuter, A. (1987). Ist der Widerstand des Patienten der Widerstand des Therapeuten? Ulm: Ulmer Textbank.
Lansky, M. R., Ed. (1992). Essential Papers on Dreams. New York and London: New York University Press.

Leuschner, W. (2006/2006). Zur Rolle «okkulter» Wahrnehmungen bei der Generierung von Trauminhalten. In JOURNAL des Psychoanalytischen Seminars Zürich, Schwerpunktthema: Fritz Morgenthaler (S. 63-80). Doppelnummer 45 (2005) / 46 (2006). Giessen: Psychosozial-Verlag.

Leuschner, W., Hau, S., Fischmann, T. (1998). Couch im Labor - Experimentelle Erforschung unbewusster Prozesse. Psyche-Zeitschrift für Psychoanalyse und ihre Anwendungen 52 , 824-849.

Morgenthaler, F. (2005 [1978]). Technik.Zur Dialektik der Psychoanalytischen Praxis. Frankfurt a.M., Syndikat; Neuauflage Giessen: Psychosozial-Verlag.

Morgenthaler, F. (2004 [1986]). Der Traum. Fragmente zur Theorie und Technik der Traumdeutung. Frankfurt a. M.: Qumran; Neuauflage Giessen: Psychosozial-Verlag.

Wittmann, L., Zander, J., Dale, A. L. (2016). Das Traumgenerierungsmodell von Ulrich Moser und Ilka von Zeppelin, Forum der Psychoanalyse 32, 39-51, DOI 10.1007/s00451-016-0224-0.

\section{Anmerkungen}

1 Diese Einleitung, die nicht zum Traumtext gehört, wird hier wiedergegeben, weil sie die Teilnehmer gehört haben.

2 Wir danken den Teilnehmerinnen des Traumseminars für ihre engagierte Mitarbeit und für die Erlaubnis, bei der Wiedergabe des Protokolls ihre Vornamen zu benützen. Das erleichtert die Lesbarkeit. Initialen des Geschlechtsnamens sind bei Teilnehmern mit speziellen Funktionen eingefügt. Beachte die Unterscheidung zwischen Michael und Michel.

3 Lutz Wittmann hatte am Anfang des Semesters in einem Vortrag das Traumgenerierungsmodell Ulrich Moser und Ilka von Zeppelin vorgestellt und am Beispiel des Traums eines kanadischen Soldaten (Wittmann et al., 2016, S. 43) illustriert.

$4 \quad$ Ralf B bezieht sich auf Konzepte der Ich-Psychologie, «wonach zu jeder Phase der Libidoentwicklung ganz bestimmte Ich-Funktionen gehören, die entwicklungsmässig paralle zu den Libidobesetzungen in den Vordergrund treten» (Morgenthaler (2004 [1986], S. 60).

$5 \quad$ Das ist die Denkleistung im manifesten Trauminhalt, was nach Freud direkt als der latente Traumgedanke aufgefasst werden kann, welcher eine harmlose Wunscherfüllung enthält. Eine solche ist hier allerdings ohne weitere Analyse nicht zu erkennen.

Für einen Überblick über die Geschichte des Subliminalisierungsverfahrens vgl. Leuschner et al. (1998).

7 Für einen ausführlichen theoretischen Überblick vgl. Binswanger (2016).

\section{Angaben zur Autorin und zum Autor}

Jeannette Widmer, MA Psych., eidgen. anerkannte Psychotherapeutin, Psychoanalytikerin PSZ in freier Praxis. 
Ralf Binswanger, Dr. med., Facharzt für Psychiatrie und Psychotherapie, Psychoanalytiker PSZ in freier Praxis. Sein psychoanalytischer Arbeitsschwerpunkt liegt in der Interpretation und eigenständigen Weiterführung der wissenschaftlichen Hinterlassenschaft von Fritz Morgenthaler in den Bereichen Dialektik, Theorie der Technik, Traum und Sexualität. 